\title{
Retropharyngeal Abscess in a Neonate After Extravasation Injury: To Drain or not to Drain?
}

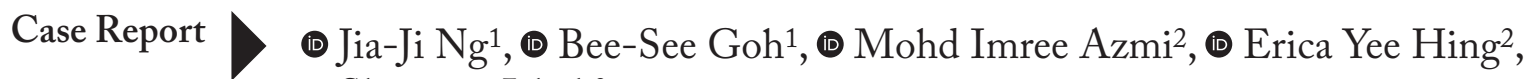 \\ (-) Shareena Ishak ${ }^{3}$ \\ ${ }^{1}$ Department of Otolaryngology, Head and Neck Surgery, University Kebangsaan Malaysia Medical Center \\ (UKMMC), Kuala Lumpur, Malaysia \\ ${ }^{2}$ Department of Radiology, University Kebangsaan Malaysia Medical Center (UKMMC), Kuala Lumpur, Malaysia \\ ${ }^{3}$ Department of Paediatric; University Kebangsaan Malaysia Medical Center (UKMMC), Kuala Lumpur, Malaysia
}

\section{ORCID ID of the authors: \\ N.J.J. 0000-0001-7209-7196; G.B.S. 0000-0002-5535-2930; M.I.A. 0000-0002-4709-1777; E.Y.H. 0000-0003-0993-6238; S.I. 0000-0003-4964-773X}

Cite this article as: $\mathrm{Ng} J \mathrm{~J}$, Goh BS, Azmi MI, Hing EY, Ishak S. Retropharyngeal Abscess in a Neonate After Extravasation Injury: To Drain or not to Drain? Turk Arch Otorhinolaryngol 2021; 59(4): 292-6.

Corresponding Author: Goh Bee See; beesee@ppukm.ukm.edu.my

Received Date: 20.04 .2021 Accepted Date: 04.07.2021

Content of this journal is licensed under a Creative Commons Attribution 4.0 International License. Available online at www.turkarchotolaryngol.net

\section{(c) (i) $\$$}

DOI: 10.4274/tao.2021.2021-4-13

Neonatal retropharyngeal abscess (RPA) is a rare and life-threatening entity. Most of the cases are idiopathic in nature. We encountered a case of RPA in a newborn secondary to extravasation injury. The presence of neck swelling with clinical deterioration following extravasation of total parenteral nutrition (TPN) infused via a peripherally inserted central catheter at the right upper limb raised the suspicion of neck abscess. This was later confirmed to be RPA based on magnetic resonance imaging of the neck. She was treated with prolonged intravenous antibiotics in the Neonatal Intensive Care Unit (NICU). Her condition gradually improved, evidenced by resolution of the collection on serial imaging. Early recognition and prompt management are crucial to reduce the morbidity and mortality from RPA.

Keywords: Retropharyngeal abscess, neonatal, extravasation of diagnostic and therapeutic materials injury, non-invasive treatment, case report

\section{Introduction}

Deep neck space infections in the pediatric age group are not frequently seen and are misdiagnosed at a rate of up to $61.8 \%$ (1). Neonatal retropharyngeal abscess is extremely rare and accounts for only $10 \%$ of all the cases in the paediatric age group (2). Nevertheless, it is an important clinical entity because of its rapid onset and lifethreatening complications such as airway obstruction, jugular vein thrombosis, mediastinal involvement, pericarditis, pneumonia, and arterial erosion (3). We report a case of retropharyngeal abscess (RPA) that occurred secondary to extravasation of total parenteral nutrition in a neonate and was successfully treated with intravenous antibiotic therapy alone. Our clinical experience with this patient highlights the benefit of the conservative antibiotic approach for RPA and the importance of multi-disciplinary team management. Though deep neck abscesses are uncommon in pediatric age group, it 
should always be considered as an important differential diagnosis in neck swelling.

\section{Case Presentation}

A female infant was delivered premature at week 32 of the gestation period with a birth weight of 1100 grams. She was born with emergency lower cesarean section due to maternal pre-eclampsia. Antenatally, her mother had history of being a Group B streptococcus (GBS) carrier. The infant was admitted to Neonatal Intensive Care Unit (NICU) from birth and was given intra-tracheal surfactant for treatment of respiratory distress syndrome (RDS). She developed necrotizing enterocolitis (NEC) on her seventh day of life and was treated with intravenous antibiotic. She was kept nil per orally and total parenteral nutrition (TPN) was commenced.

A peripherally inserted central catheter (PICC) was inserted at the right cubital fossa on day 16 of her life. After starting TPN, there was immediate right neck swelling with facial edema. Chest radiograph (CXR) showed the catheter tip at the junction of right subclavian and brachiocephalic vein, and there was no evidence of distal migration of the catheter (Figure 1). This incident was treated as extravasation of TPN and PICC was removed. The neck swelling and facial edema gradually subsided after one week of intravenous antibiotic therapy. Coagulase-negative staphylococci (CoNS) was isolated from the PICC tip.

However, the right neck swelling recurred after completion of intravenous antibiotic therapy. The non-fluctuant, nontender swelling was seen mainly over the right submental and submandibular area with normal skin color. There was no subcutaneous emphysema palpable. She had developed multiple apneic episodes requiring Bi-level Positive Airway Pressure (BiPAP) support. She also had fever of 37.9 degree Celsius and her heart rate was persistently tachycardic ranging from 180-200 beats per minute during this period.

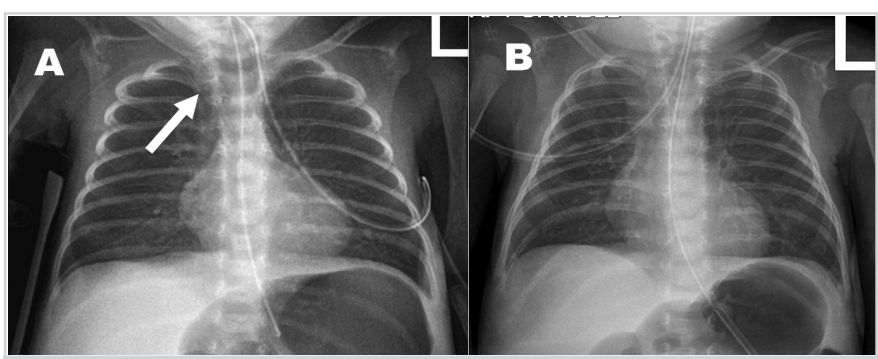

Figure 1a. Chest radiographs (CXR) taken post-right percutaneous insertion of peripherally inserted central catheter (PICC). The white arrow shows the tip of the PICC at the junction of right subclavian vein and right brachiocephalic vein with no fracture of PICC or pneumothorax. Tip of nasogastric tube was seen in the stomach.b. CXR taken when patient developed right neck swelling. The PICC was removed prior to CXR. There was no abnormality in the CXR to suspect retropharyngeal collection
However, there were no hypotensive episodes. Her blood investigations showed a white cell count of $27.3 \times 10^{\wedge} 9 / \mathrm{L}$ which was neutrophils predominant $(92.9 \%)$ and an increased C-reactive protein level of $3.52 \mathrm{mg} / \mathrm{dl}$. As the repeat chest radiograph did not reveal any obvious abnormality, an ultrasound of the right neck was performed. The ultrasound showed presence of a hypoechoic collection seen posterior to the right carotid sheath, with presence of echogenic debris suggestive of an RPA (Figures $2 \mathrm{a}$ and $\mathrm{b}$ ). The collection had crossed the midline retropharyngeally to the left, posterior to the left carotid sheath. Anteriorly, the collection had also displaced the right internal jugular vein (IJV) and the common carotid artery which remained patent. Magnetic resonant imaging (MRI) of the neck confirmed the findings of the ultrasound examination. The collection was seen as hypointense signal on T1W1, hyperintense signal on T2WI and rim enhancement on post contrast (Figures $3 a$ and $b$ ). A diagnosis of RPA was made, and intravenous antibiotic therapy was re-commenced. Blood culture then grew CoNS which was sensitive to teicoplanin and rifampicin.

She was referred to the Otolaryngology team in view of the possibility that the RPA may cause upper airway obstruction. A repeat ultrasound performed one week after commencement of treatment showed non-resolution of the collection. It partially compressed the right IJV and displaced the oesophagus to the left (Figures $2 \mathrm{c}$ and $\mathrm{d}$ ).

Though there was a soft diffuse right submandibular swelling, she did not have any symptoms of upper airway obstruction. There was no stridor and no bulge seen at the posterior

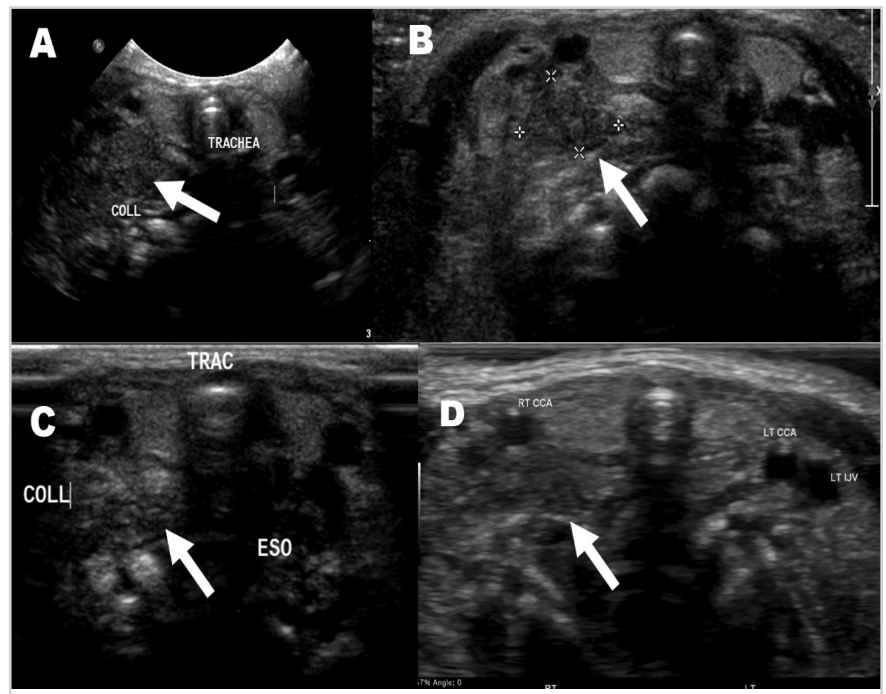

Figure 2.Neckultrasound:a-b. white arrow, therewas a heterogeneous hypoechoic collection in the right neck region posterior to the right internal jugular vein (IJV) and lateral to the oesophagus measuring $1.0 \times 2.5 \times 1.6 \mathrm{~cm}(\mathrm{APxWxCC})$; $\mathrm{c}-\mathrm{d}$. white arrow, repeated ultrasound of the right neck region one week following treatment showed nonresolution of the previously seen collection. This collection did not cause airway or vascular compression 
pharyngeal wall. She remained well with satisfactory weight gain. The workup for primary immunodeficiency which included $\mathrm{T}$ and $\mathrm{B}$ cell and natural killer cell profiles were unremarkable. In view of the clinical improvement, a multidisciplinary team decision was made to continue the medical therapy with serial imaging and blood parameter monitoring. A neck ultrasound which was performed three weeks after the antibiotic therapy revealed presence of phlegmon at the previous collection site with suspicion of right IJV thrombus formation. An MRI/Magnetic resonance venography (MRV) after six weeks of therapy (combination of intravenous teicoplanin and rifampicin) showed resolution of the retropharyngeal abscess and right IJV thrombosis with presence of collaterals (Figures $3 \mathrm{c}$ and $\mathrm{d}$ ).

She was discharged at corrected gestation of 42 weeks and weight of 2670 grams. A repeat ultrasound performed six weeks after completion of intravenous antibiotic therapy revealed resolution of collection with short segment right IJV stenosis.

\section{Discussion}

While deep neck infections can be difficult to diagnose and treat, they are more challenging among the pediatric age group. Deep neck infections can be categorized based

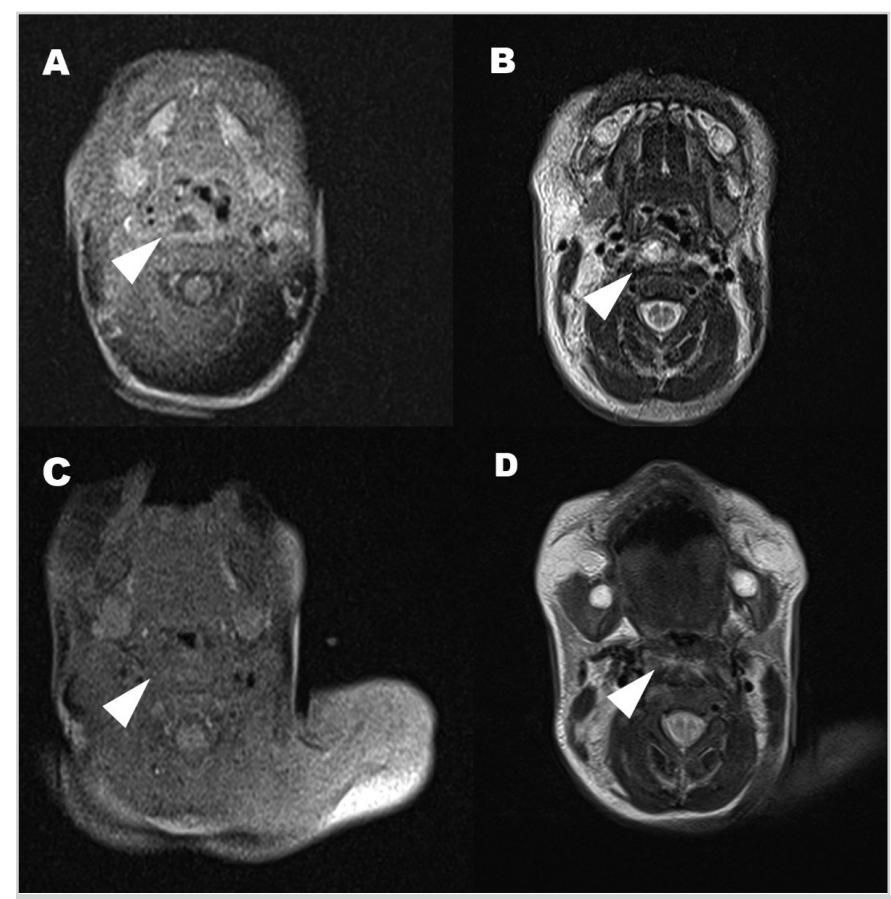

Figure 3. Magnetic resonant imaging (MRI) of the neck: $a-b$. white arrowhead, the initial MRI of the neck showed the previous collection seen on ultrasound. It showed (b, white arrowhead) T2WI hyperintense signal and (a, white arrowhead) rim enhancement measuring approximately $0.5 \times 2.0 \times 1.3 \mathrm{~cm}(\mathrm{APxWxCC})$ in widest diameter in post contrast in the right retropharyngeal space. Repeat MRI one month after treatment showed resolution of the collection. There was no longer (c) rim enhancing collection or (d) T2WI hyperintense signal on their anatomical site; namely as retropharyngeal, parapharyngeal, and peritonsillar abscesses. RPA is the most common, constituting $66.7 \%$ of deep neck infections in the pediatric age group (4).

The retropharyngeal space is a potential space between the visceral layer and deep layer of the cervical fascia. This space contains large numbers of lymph nodes, especially in children under five years of age. The retropharyngeal space lymph nodes drain the nasal cavity, the nasopharynx, the paranasal sinuses, as well as the soft palate. Therefore, upper respiratory tract infections are the leading cause of RPA in the pediatric age group (2). Dental infections or congenital anomalies may also be a source of RPA formation. On the other hand, trauma and foreign body ingestion are more likely in adult patients. Staphylococcus aureus is the most common microorganism isolated in pediatric RPA $(1,3)$. It is also important to note that children from lower socioeconomical background are more at risk as they may not be able to seek treatment in time and might need more invasive approaches (4).

As for neonates, deep neck abscesses are rare. Male neonates are more at risk, with a male to female ratio of $2: 1$ (3). Though most of the cases are idiopathic, there are some known predisposing factors, such as history of intubation, nasopharyngeal CPAP, multiple laryngoscopies and even application of suction catheter (3). Presence of congenital abnormalities such as remnant of third branchial arch or cystic hygroma should also be investigated and treated if a patient develops recurrent deep neck abscesses (3).

Clinical presentation in neonates may differ from older children. Though fever is common in childhood RPA, it is usually absent in the neonate. The dangers of a RPA are airway obstruction and spread of infection to involve the carotid sheath and/or the mediastinum (3). As a result, the affected neonate may develop stridor and/or respiratory distress, poor feeding, hoarseness, and weak cry (3). Trismus, drooling, retropharyngeal bulge, and acute respiratory compromise are less common and require immediate medical attention. It may be misdiagnosed as up to $40 \%$ of pediatric patients may have no visible neck swelling or restricted neck movement on presentation $(1,5)$. In our case report, our patient was a preterm neonate with a predisposing factor of nasal CPAP use. Nevertheless, extravasation of TPN was more likely to have caused the neck swelling which later got infected.

Extravasation injury is caused by non-intentional leakage of infused intravenous fluid into the surrounding soft tissue, leading to local tissue reaction or damage (6). Extravasation has been shown to occur in up to $70 \%$ of neonates, although tissue damage and skin necrosis is much less common (7). Intravenous lines are often needed for long periods in neonates as a means to provide nutrition and medication. As neonatal veins are small and fragile, this increases their susceptibility to extravasation injury (7). Parenteral nutrition 
has been one of the most common implicated causes of extravasation injury (7). Total parenteral nutrition is a vesicant and hyperosmolar solution that is rich in lipids, amino acids, dextrose, vitamins, electrolytes and trace elements, and has the potential to cause varying degrees of deep tissue damage (7). If extravasation of this hyperosmolar solution occurs, it can cause severe damage, such as skin necrosis, skin infections with abscess, or even necrotizing fasciitis. Although the exact mechanism of tissue injury is unclear, the proposed mechanism has been suggested to be related to the hyperosmolarity, acidic $\mathrm{pH}$ and toxic effect of local ions in parenteral nutrition $(6,7)$. Extravasation injury can be treated conservatively by local topical application and antidote administration, or with the saline washout technique (6). There is no consensus on the best treatment for extravasation injury and the importance of prevention has not been sufficiently emphasized.

As deadly complications may arise from deep neck abscesses, urgent surgical drainage has been advocated by many surgeons $(8,9)$. This is true in adults, as the infection can spread easily along the fascial plane. In children, however, infection is usually confined to the lymph node and spreads only when the lymph node ruptures. With this clinical relevance, studies were done to challenge the conventional method of surgical drainage $(5,10)$. The wait and watch method with high dose intravenous antibiotics has been shown to be as effective as the drainage method without increasing morbidity and mortality rates or without increasing the length of stay in the hospital $(5,10)$. It is also worth noting that no pus in up to $21 \%$ of the patients who were admitted for surgical management (8).

In cases with airway complications, severe neck movement restriction, bulging of pharyngeal wall, or mediastinitis with significant medical co-morbidities, surgical drainage of the RPA, transoral or transcervical, is more advisable (5). Page et al. (8) proposed positive predictive factors, such as duration of illness of more than two days, history of antibiotic usage and computed tomography of the lesion of more than $2 \mathrm{~cm}^{2}$, for advocating surgical drainage.

\section{Conclusion}

Early recognition of neonatal RPA is important, and appropriate investigation should be carried out promptly to prevent severe life-threatening complications. RPA secondary to extravasation of TPN is relatively unusual but may cause infection as illustrated in this case report. Fortunately, early recognition and radiologic examination, together with the administration of high dose intravenous antibiotics halted the progression of the disease with complete resolution without any surgical intervention.

\section{Authorship Contributions}

Conception: N.J.J., G.B.S., Design: N.J.J., G.B.S., Supervision: N.J.J., G.B.S., E.Y.H., S.I., Data Collection and/or Processing: N.J.J., G.B.S., M.I.A., Analysis and/or Interpretation: N.J.J., G.B.S., M.I.A., E.Y.H., S.I., Literature Review: N.J.J., G.B.S., M.I.A., E.Y.H., S.I., Writing: N.J.J., G.B.S., M.I.A., S.I., Critical Review: N.J.J., G.B.S., M.I.A., E.Y.H., S.I.

Informed Consent: Consent was obtained from the patient's parents. Her anonymity was preserved throughout the case report.

Peer-review: Externally peer-reviewed.

Conflict of Interest: No conflict of interest was declared by the authors.

Financial Disclosure: The authors declared that this study received no financial support.

\section{Main Points}

- Extravasation of total parenteral nutrition in the neonate is one of the rare causes of neonatal retropharyngeal abscess.

- Early recognition of the disease and early administration of high dosage of antibiotics will halt the progression of the disease without surgical intervention.

- The wait-and-watch approach with intravenous antibiotics is as effective as the surgical drainage method without increasing the risks of morbidity and mortality in selected cases.

\section{References}

1. Poonit ND, Zhou YY, Xu DF, Qiu XH, Chen YF, Li ZJ, et al. Retropharyngeal abscess in children: a retrospective study. Ann Pediatr Res 2019; 3: 1027. [Crossref]

2. Huang CM, Huang FL, Chien YL, Chen PY. Deep neck infections in children. J Microbiol Immunol Infect 2017; 50: 62733. [Crossref]

3. Mutlu M, Dereci S, Aslan Y. Deep neck abscess in neonatal period: case report and review of literature. Int J Pediatr Otorhinolaryngol 2014; 78: 577-82. [Crossref]

4. Angajala V, Hur K, Jacobson L, Hochstim C. Geographic health disparities in the Los Angeles pediatric neck abscess population. Int J Pediatr Otorhinolaryngol 2018; 113: 134-9. [Crossref]

5. Wong DKC, Brown C, Mills N, Spielmann P, Neeff M. To drain or not to drain - management of pediatric deep neck abscesses: a case-control study. Int J Pediatr Otorhinolaryngol 2012; 76: 18103. [Crossref] 
6. Mas V, Simon AL, Presedo A, Mallet C, Ilharreborde B, Jehanno P. Upper limb extravasation of cytotoxic drugs: results of the saline washout technique in children. J Child Orthop 2020; 14: 230-5. [Crossref]

7. Restieaux M, Maw A, Broadbent R, Jackson P, Barker D, Wheeler B. Neonatal extravasation injury: prevention and management in Australia and New Zealand-a survey of current practice. BMC Pediatr 2013; 13: 34. [Crossref]

8. Page NC, Bauer EM, Lieu JEC. Clinical features and treatment of retropharyngeal abscess in children. Otolaryngol Head Neck Surg 2008; 138: 300-6. [Crossref]
9. Kirse DJ, Roberson DW. Surgical management of retropharyngeal space infections in children. Laryngoscope 2001; 111: 1413-22. [Crossref]

10. Cramer JD, Purkey MR, Smith SS, Schroeder JW. The impact of delayed surgical drainage of deep neck abscesses in adult and pediatric populations. Laryngoscope 2016; 126: 1753-60. [Crossref] 